\title{
REVERSE CONVEX PROBLEMS: AN APPROACH BASED ON OPTIMALITY CONDITIONS
}

\author{
IDER TSEVEENDORJ
}

Received 9 February 2006; Accepted 10 February 2006

We present some results concerning reverse convex problems. Global optimality conditions for the problems with a nonsmooth reverse convex constraint are established and convergence of an algorithm in the case of linear program with an additional quadratic reverse convex constraint is studied.

Copyright (c) 2006 Ider Tseveendorj. This is an open access article distributed under the Creative Commons Attribution License, which permits unrestricted use, distribution, and reproduction in any medium, provided the original work is properly cited.

\section{Introduction}

In the present paper we consider the nonconvex problem

$$
\begin{gathered}
\operatorname{minimize} f(x), \\
\text { subject to } x \in S, \\
g(x) \geq 0,
\end{gathered}
$$

where $f, g: \mathbb{R}^{n} \rightarrow \mathbb{R}$ are convex continuous functions and $S$ is a nonempty, convex compact in $\mathbb{R}^{n}$.

Such problems have many practical and theoretical applications in telecommunication, mechanics, engineering design, economics, and other fields (see [1, 2, 21], etc.) and have been studied actively over the last four decades (see, e.g., [9, 19] and their references). In addition to these direct applications, $(R P)$ may appear as subproblems in more difficult nonconvex problems $[5,17,20]$. Also it is known that reverse convex problems and the well-known convex maximization (concave minimization) problems are dual to each other and the latter has an abundance of applications [12, 19].

In the literature, this problem is known as the minimization problem with a "hole" [3], the reverse convex problem (see $[7,11]$, etc.), the problem over complements of convex sets [13], the canonical d.c. programming problem (see $[8,18]$, etc.), and anticonvex 
programs [12]. All these various expressions are used to describe the constraint $g(x) \geq 0$ that creates difficulties and destroys the convexity of the problem.

Throughout this paper we will call the problem "the reverse convex."

A good deal of literature exists on methods for solving problem $(R P)$. Our focus in this paper is on an approach based on global optimality conditions.

The purpose of this paper is to establish necessary and sufficient optimality conditions for nonsmooth reverse convex problems $(R P)$ and to study some convergence properties of an algorithm in the case of LP with an additional quadratic reverse convex constraint.

The present paper is organized as follows. First, we derive necessary and sufficient optimality conditions for nonsmooth reverse convex problems in Section 2. Then in order to be self-contained in Section 3, we recall from $[15,16]$ an algorithm for solving $(R P)$ and some of its convergence properties. Finally, Section 4 is devoted to the LP problem with an additional quadratic reverse convex constraint. As a result of this section we prove a proposition which plays an essential role in providing convergence of the algorithm.

Our notation is fairly standard in convex analysis. $\operatorname{By} \partial g(\cdot), \operatorname{cl}(D), \operatorname{co}(D)$ we denote the subdifferential, the closure, and the convex hull, respectively.

\section{Necessary and sufficient optimality conditions}

We will use further notation:

$$
\begin{gathered}
D=\left\{x \in \mathbb{R}^{n} \mid x \in S, g(x) \geq 0\right\}, \\
F(z)=\left\{x \in \mathbb{R}^{n} \mid x \in S, f(x) \leq f(z)\right\}, \\
N(S, y)=\left\{y^{*} \in \mathbb{R}^{n} \mid\left\langle y^{*} x-y\right\rangle 0, \forall x \in S\right\} .
\end{gathered}
$$

In what follows, we will require the following two assumptions to hold even if this is not always mentioned explicitly.

Assumption 2.1. There exists a finite solution of problem $(R P)$, that is,

$$
\inf \{f \mid D\}>-\infty, \quad \exists x_{0} \in D: f\left(x_{0}\right) \neq+\infty .
$$

Assumption 2.2. At a solution of problem $(R P)$ the reverse convex constraint is active, that is,

$$
\operatorname{Argmin}(R P) \cap\{x \mid g(x)>0\}=\varnothing,
$$

where by "Argmin" we denote a set of solutions.

The latter assumption is quite standard for $(R P)$, which means that the reverse convex constraint $g(x) \geq 0$ is essential.

THeORem 2.3. If for a reverse convex problem (RP) (2.3) holds (Assumption 2.2), and if its feasible point $z$ solves problem $(R P)$ globally, then

$$
\begin{gathered}
\forall y \quad \text { s.t. } g(y)=0, \\
\partial g(y) \cap N(F(z), y) \neq \varnothing .
\end{gathered}
$$


Proof. Let us assume that $z$ solves $(R P),(2.3)$ holds, but (2.4) does not hold. Then there is $y$ such that $g(y)=0$ and

$$
\partial g(y) \cap N(F(z), y)=\varnothing
$$

Two given sets do not intersect when any element of one of them does not belong to the second; in our case:

$$
\text { for any } y^{*} \in \partial g(y) \text { we have } y^{*} \notin N(F(z), y) \text {. }
$$

The latter implies $\exists x \in F(z):\left\langle y^{*} x-y\right\rangle>0$.

In other words, what we assumed means that there are $y, x$ such that $g(y)=0, f(x) \leq$ $f(z), x \in S$ satisfying

$$
\left\langle y^{*}, x-y\right\rangle>0, \quad \forall y^{*} \in \partial g(y) .
$$

By the convexity of function $g(\cdot)$ one obtains

$$
0<\left\langle y^{*}, x-y\right\rangle \leq g(x)-g(y)=g(x) .
$$

Then $f(x) \leq f(z), x \in S$ together with (2.8) implies that $x$ also solves $(R P)$ since $z$ is a solution to $(R P)$. It contradicts (2.3) because of $g(x)>0$ in (2.8). This contradiction completes the proof.

Example 2.4. We consider a two-dimensional problem $(R P)$ (see Figure 2.1), with

$$
\begin{gathered}
f(x)=\left(x_{1}-\frac{1}{4}\right)^{2}+\left(x_{2}-\frac{1}{4}\right)^{2}, \\
g(x)=2\left|x_{1}\right|+\left|x_{2}\right|-1, \\
S=\Pi=\left\{x \in \mathbb{R}^{2} \mid-\frac{1}{2} \leq x_{1} \leq \frac{1}{4},-\frac{3}{4} \leq x_{2} \leq \frac{5}{4}\right\} .
\end{gathered}
$$

Here the reverse convex constraint function is the piecewise linear (nonsmooth) function. Similar domains are encountered frequently in optimization problems of economics [21].

It is not difficult to see that at point $z=(-9 / 20,-1 / 10)^{\top}$ the first-order optimality condition:

$$
\exists \mu \geq 0, \exists z^{*} \in \partial g(z) \quad\left\langle\nabla f(z)-\mu z^{*}, x-z\right\rangle \geq 0, \quad \forall x \in \Pi,
$$

holds since at $z$ function $g(\cdot)$ is differentiable $\partial g(z)=\{\nabla g(z)\}$ and $\nabla f(z)-\mu \nabla g(z)=0$. The number of such stationary points $z$ where the first-order optimality condition holds could be very huge in nonconvex optimization and we do not know which one fails to be a global solution. To answer this question we use a necessary global optimality condition. 


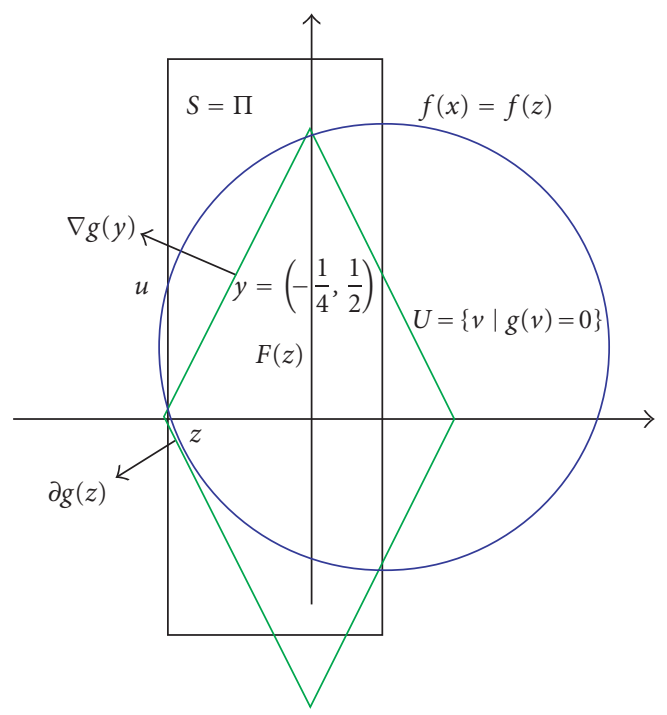

Figure 2.1. The necessary condition (Example 2.4).

Let us try to use (2.4) to get the answer. We consider points

$$
\begin{aligned}
& y=\left(-\frac{1}{4}, \frac{1}{2}\right)^{\top} \text { satisfying } g(y)=0, \\
& u=\left(-\frac{9}{20}, \frac{1}{2}\right)^{\top} \text { satisfying } f(u) \leq f(z), \quad u \in \Pi .
\end{aligned}
$$

It is easy to see that at point $y$ the subdifferential of function $g(\cdot)$ consists of a unique element (gradient) and we have $\langle\nabla g(y), u-y\rangle=2 / 5>0$. According to the necessary optimality conditions we can assert that $z$ is not a global solution to the considered problem.

The next theorem gives us a sufficient condition.

Theorem 2.5. Assume that for a feasible point $z$ of problem (RP)

$$
-\infty \leq \inf \left(g, \mathbb{R}^{n}\right)<g(z)=0
$$

is true, and in addition suppose that

$$
\begin{aligned}
& \forall y \in S \quad \text { s.t. } g(y)=0, \quad \exists y^{*} \in \partial g(y), \\
& \exists h=h\left(y, y^{*}\right) \in S, \quad\left\langle y^{*}, h-y\right\rangle>0 .
\end{aligned}
$$

Then a sufficient condition for the point $z$ to be a global solution to $(R P)$ is

$$
\begin{gathered}
\forall y \quad \text { s.t. } g(y)=0, \\
\partial g(y) \cap N(F(z), y) \neq \varnothing .
\end{gathered}
$$


Proof. Suppose that the assumptions of the theorem are satisfied and (2.14) holds, but $z$ is not a global solution to $(R P)$.

So, there is $w$ such that

$$
w \in S, \quad g(w) \geq 0, \quad f(w)<f(z) .
$$

First of all, we show an existence of $u$ such that

$$
u \in S, \quad g(u)>0, \quad f(u)<f(z)
$$

It is clear that if $g(w)>0$, then $u=w$.

Thus we consider only the case of $g(w)=0$. Due to the continuity of function $f(\cdot)$ there is a neighborhood $W$ of the point $w$, for which we have

$$
f(x)<f(z), \quad \forall x \in W
$$

According to (2.13) we also have

$$
\exists w^{*} \in \partial g(w), \exists h \in S \quad\left\langle w^{*}, h-w\right\rangle>0 .
$$

Then because of the convexity of function $g(\cdot)$ for $\alpha \in] 0,1[$, and $x(\alpha)=\alpha h+(1-\alpha) w$ we arrive at

$$
g(x(\alpha))-g(w) \geq\left\langle w^{*}, x(\alpha)-w\right\rangle=\alpha\left\langle w^{*}, h-w\right\rangle>0 .
$$

This means

$$
g(x(\alpha))>0, \quad x(\alpha) \in S, \quad \alpha \in] 0,1[.
$$

So, for the sufficiently small positive number $\alpha_{0}$ it is evident that $x\left(\alpha_{0}\right) \in W$; and according to (2.17) we have

$$
f\left(x\left(\alpha_{0}\right)\right)<f(z)
$$

Therefore, $u=x\left(\alpha_{0}\right)$ satisfies (2.16).

On the other hand (2.12) implies the existence of $v$ such that $g(v)<g(z)=0$.

Due to the continuity of $g(\cdot)$ and inequalities $g(v)<0<g(u)$, the open interval connecting two points $v$ and $u$ :

$$
y(\beta)=\beta v+(1-\beta) u, \quad 0<\beta<1,
$$

intersects the level set $g(x)=0$ at some point $y^{0}=y\left(\beta_{0}\right),\left(0<\beta_{0}<1\right)$.

Now we show that any subgradient $y_{0}^{*} \in \partial g\left(y^{0}\right)$ does not belong to $N\left(F(z), y^{0}\right)$, that is, for all $y_{0}^{*} \in \partial g\left(y^{0}\right)$ there is $u \in F(z)$ satisfying $\left\langle y_{0}^{*} u-y^{0}\right\rangle>0$. 
By replacing $u$ by its expression from (2.22) we have for $u \in F(z)$ and for all $y_{0}^{*} \in$ $\partial g\left(y^{0}\right)$

$$
\begin{aligned}
\left\langle y_{0}^{*}, u-y^{0}\right\rangle & =\left\langle y_{0}^{*}, \frac{y^{0}-\beta_{0} v}{1-\beta_{0}}-y^{0}\right\rangle=\frac{\beta_{0}}{\beta_{0}-1}\left\langle y_{0}^{*}, v-y^{0}\right\rangle \\
& \geq \frac{\beta_{0}}{\beta_{0}-1}\left(g(v)-g\left(y^{0}\right)\right)=\frac{\beta_{0}}{\beta_{0}-1} g(v)>0,
\end{aligned}
$$

that means $y_{0}^{*} \notin N\left(F(z), y^{0}\right)$. Hence $\partial g\left(y^{0}\right) \cap N\left(F(z), y^{0}\right)=\varnothing$ is contradicting (2.14). The theorem now follows.

Remark 2.6. The core expression of both necessary and sufficient optimality conditions

$$
\partial g(y) \cap N(F, y) \neq \varnothing
$$

could be written as

$$
\begin{gathered}
\exists y^{*} \in \partial g(y), \\
\left\langle y^{*}, x-y\right\rangle \leq 0, \quad \forall x \in F .
\end{gathered}
$$

Its checking requires first solving the problem

$$
\begin{gathered}
\operatorname{maximize}\left\langle y^{*}, x\right\rangle, \\
\text { subject to } x \in F,
\end{gathered}
$$

and then verifying the inequality below on its solution $x(y)$ :

$$
\left\langle y^{*} x(y)-y\right\rangle \leq 0
$$

The following example shows how one could use sufficient optimality conditions obtained to prove the global optimality of some point.

Example 2.7. We continue to consider the problem from Example 2.4. Suppose that we found a stationary point one way or another; for instance in our case (see Figure 2.2) $z=(1 / 4,1 / 2)^{\top}$. To assert its global optimality we have to use a sufficient global optimality condition. Let us try to use Theorem 2.5. According to Theorem 2.5, before checking (2.14) one has to check assumption (2.13).

It is quite evident that for any point $y$ of the level set $g(y)=0$ there is a subgradient $y^{*} \in \partial g(y)$ such that $\left\langle y^{*}, h-y\right\rangle>0$, where $h$ is one of the vertices of the box $\Pi$, that is, assumption (2.13) holds in this case.

Now we divide the level set $U=\{v \mid g(v)=0\}$ into four parts $U=U^{11} \cup U^{10} \cup U^{00} \cup$ $U^{01}$, corresponding to four orthants.

Notice that the solution to problem

$$
\begin{array}{r}
\operatorname{maximize}\left\langle v^{*}, x\right\rangle, \\
\text { subject to } x \in F(z),
\end{array}
$$




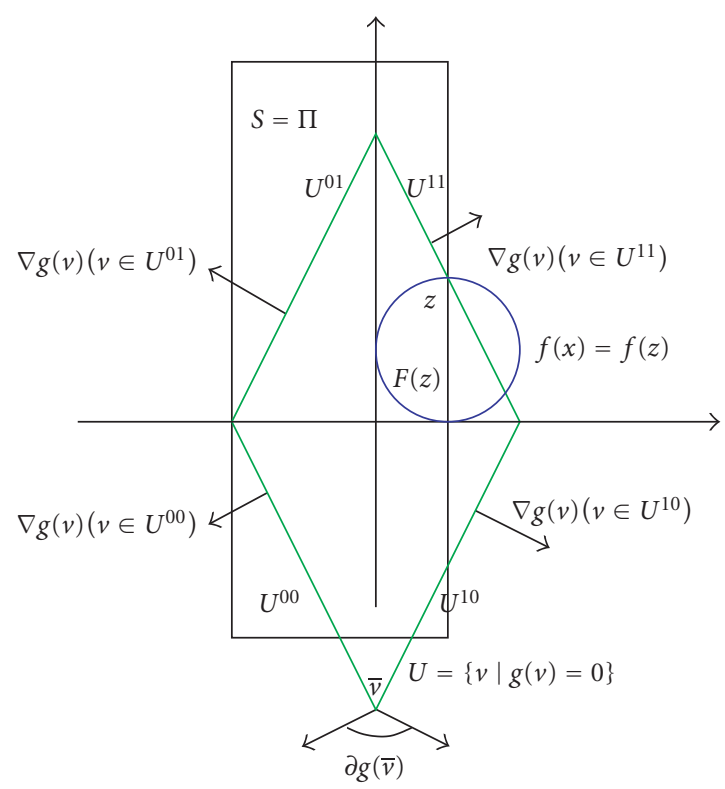

Figure 2.2. The sufficient condition (Example 2.7).

where $v^{*} \in \partial g(v)$, could be written as

$$
u(v)= \begin{cases}\left(\frac{1}{4}, \frac{1}{2}\right)^{\top}, & \text { if } v \in U^{11} \\ \left(\frac{1}{4}, 0\right)^{\top}, & \text { if } v \in U^{10} \\ \left(\frac{1}{4}-\frac{1}{2 \sqrt{5}}, \frac{1}{4}-\frac{1}{4 \sqrt{5}}\right)^{\top}, & \text { if } v \in U^{00} \\ \left(\frac{1}{4}-\frac{1}{2 \sqrt{5}}, \frac{1}{4}+\frac{1}{4 \sqrt{5}}\right)^{\top}, & \text { if } v \in U^{01}\end{cases}
$$

Then it is not difficult to see that for all $v$ such that $g(v)=0$ we have

$$
\left\langle v^{*}, u(v)-v\right\rangle \leq 0 \text {. }
$$

In other words, there is no point on the level set $\{v \mid g(v)=0\}$ where the sufficient global optimality condition is violated. Thus, according to Theorem $2.5, z$ is a global solution to the considered problem.

Remark 2.8. Similar optimality conditions for reverse convex problems $(R P)$ were first obtained by Strekalovsky $[13,14]$. Therein the author used the following assertion:

$$
\forall y: g(y)=0, \quad \partial g(y) \subset N(F(z), y),
$$


instead of our (2.4) and (2.14) from Theorems 2.3 and 2.5, respectively. One can see the difference between our $\partial g(y) \cap N(F(z), y) \neq \varnothing$ and (2.31) (i.e., the difference between checking the nonemptyness of the intersection of two convex sets and the inclusion of one convex set in the other) since the latter is generally more difficult to check in practice.

But in the case of the differentiable function $g(\cdot)$ there is no difference between them (inclusion, intersection) since the subdifferential $\partial g(\cdot)$ consists of a single element $\nabla g(\cdot)$ and it intersects a normal cone if and only if it itself lies in the normal cone.

\section{Algorithm and its convergence properties}

In the following sections in addition we will assume that function $g(\cdot)$ is quadratic; $g(x)=$ $(1 / 2)\langle Q x, x\rangle-\gamma$ where $Q=Q^{\top}$ and $Q>0$ (positive definite) and $\gamma>0$.

In order to be self-contained, let us recall the main lines of an algorithm for finding a global solution to the reverse convex problem $(R P)$ based on optimality conditions like (2.4), (2.14), (2.31). The algorithm was proposed in [15] and its convergence was proven in $[16]$.

Given a point $x^{0}$ such that $x^{0} \in S, g\left(x^{0}\right) \geq 0$, and $k=0$.

The algorithm consists of the following steps.

Algorithm 3.1.

Step 1. Let $z^{k}$ be a local minimum (or a stationary point) of $(R P)$ with starting point $x^{k}$.

Step 2. Approximate the level set $\{y \mid g(y)=0\}$ by $R_{k}=R\left(z^{k}\right)=\left\{v^{1}, v^{2}, \ldots, v^{N_{k}} \mid g\left(v^{i}\right)=0\right.$, $\left.i=1, \ldots, N_{k}\right\} ; i:=1$.

Step 3. Solve $u^{i}=\operatorname{argmax}\left\{\left\langle\nabla g\left(v^{i}\right), x\right\rangle \mid x \in F\left(z^{k}\right)\right\}$.

Step 4. Solve $w^{i}=\operatorname{argmax}\left\{\left\langle\nabla g(y), u^{i}-y\right\rangle \mid g(y)=0\right\}$.

Step 5. If $\left.\left(\left\langle\nabla g\left(w^{i}\right), u^{i}-w^{i}\right\rangle\right\rangle 0\right)$, then $x^{k+1}:=u^{i}, k:=k+1$, go to Step 1 ; ${ }^{*}$ new local search $* /$.

Step 6. Else if $\left(i<N_{k}\right)$, then $i:=i+1$ go to Step 3 .

Step 7. Else STOP; $/ * z^{k}$ is the solution related to $R_{k} * /$.

Remark 3.2. Assume that for some index $i$ one has $\left\langle\nabla g\left(w^{i}\right), u^{i}-w^{i}\right\rangle>0$ and therefore $x^{k+1}:=u^{i}$. Then $x^{k+1} \in S, f\left(x^{k+1}\right) \leq f\left(z^{k}\right)$ since $x^{k+1} \in F\left(z^{k}\right)$, and $g\left(x^{k+1}\right)>0$ since $g\left(x^{k+1}\right)-g\left(w^{i}\right) \geq\left\langle\nabla g\left(w^{i}\right), u^{i}-w^{i}\right\rangle>0$.

So, the algorithm generates a sequence $\left\{z^{k}\right\}$ of stationary points such that

$$
f\left(z^{k+1}\right) \leq f\left(x^{k+1}\right) \leq f\left(z^{k}\right), \quad \forall k=0,1,2, \ldots
$$

In the appendix, we remind the proof of convergence of a theoretical method given in [16]. It is not difficult, under assumptions (a1)-(a4) below, to specialize the theoretical method into the above practical algorithm.

(a1) There is a method for finding a local solution (or stationary point) to problem $(R P)$. 
(a2) There is a method for solving the problem

$$
\begin{gathered}
\text { maximize }\left\langle\nabla g\left(v^{i}\right), x\right\rangle, \\
\text { subject to } x \in S, \\
f(x) \leq f\left(z^{k}\right) .
\end{gathered}
$$

(a3) There is a method for solving the problem

$$
\begin{gathered}
\operatorname{maximize}\left\langle\nabla g(y), u^{i}-y\right\rangle, \\
\text { subject to } g(y)=0 .
\end{gathered}
$$

(a4) One can construct an approximation of the level set $\{y \mid g(y)=0\}$ that is a "resolving set" according to Definition 3.3 below.

In general, in the case of unsuccessful approximations used in Step 2, the solution found by the algorithm could fail to be a global solution. Therefore we introduce the notion of the "resolving set" for the approximations that guarantees the globality of a solution obtained.

Definition $3.3[15,16]$. An approximation

$$
R=\left\{v^{1}, v^{2}, \ldots, v^{N} \mid g\left(v^{i}\right)=0, i=1, \ldots, N\right\}
$$

is called a "resolving set" if the fact that $z$ is not a global solution to $(R P)$ implies

$$
\max _{i}\left\{\left\langle\nabla g\left(w^{i}\right), u^{i}-w^{i}\right\rangle \mid i \in\{1,2, \ldots, N\}\right\}>0 .
$$

Example 3.4. We come back to the problem from Examples 2.4 and 2.7. It is not difficult to see that approximation

$$
R=\left\{\left(\frac{1}{4}, \frac{1}{2}\right)^{\top},\left(-\frac{1}{4}, \frac{1}{2}\right)^{\top},\left(-\frac{1}{4},-\frac{1}{2}\right)^{\top},\left(\frac{1}{4},-\frac{1}{2}\right)^{\top}\right\}
$$

is a "resolving set."

Let us discuss the assumptions. In our opinion, assumptions (a1), (a2), (a3) are relatively easier than (a4).

Nowadays, usable local search methods have been designed $[1,2,16]$ for reverse convex problems despite its being NP-hard.

The problem in (a2) is convex if function $f(\cdot)$ and set $S$ are assumed to be convex.

With regard to (a3), in general, it is a nonconvex problem but the problem can be solved analytically in some simple cases. For instance [15], in the quadratic case, that is, $g(x)=(1 / 2)\langle Q x, x\rangle-\gamma$ where $Q=Q^{\top}$ and $Q>0$ (positive definite) and $\gamma>0$, point

$$
w^{i}=\left(\frac{2 \gamma}{\left\langle Q u^{i}, u^{i}\right\rangle}\right)^{1 / 2} u^{i}
$$

solves the problem of (a3). 
Finally, as regards (a4) there is no approximation proved to be a "resolving set" except some simple cases in [4]. In practice we have used the algorithm without the proof that approximations are "resolving sets." For instance, in $[10,15]$ the approximation consists of some deterministic points issuing from the problem. The authors of [6] to solve the well-known multiknapsack problem have proposed randomly generating level set points where the number of points is limited by a tabu list to prevent the same level set area from being re-examined. In all cases, the experiments showed that the algorithm is promising, but it is always important to prove that an approximation used is a "resolving set." The next section is devoted to the question of how to prove it.

\section{Example of "resolving set"}

We consider the linear programming problem with an additional reverse convex quadratic constraint:

$$
\begin{gathered}
\text { minimize }\langle d, x\rangle, \\
\text { subject to } A x \leq b, \\
g(x)=\frac{1}{2}\langle Q x, x\rangle-\gamma \geq 0,
\end{gathered}
$$

where $d, x \in \mathbb{R}^{n}, A^{\top}=\left(a^{1}, a^{2}, \ldots, a^{m}\right)$ is an $(n \times m)$-matrix, $b \in \mathbb{R}^{m}, Q=Q^{\top}$ and $Q>0$ (positive definite) $(n \times n)$-matrix, and $\gamma>0$. We assume that here the polytope in the constraint is full dimensional and bounded.

Proposition 4.1. Suppose that assumption (2.13) holds, which may be written in terms of (LRCP) as follows:

$$
\begin{gathered}
\forall y \text { such that } g(y)=0 \text { and } A y \leq b \\
\text { there exists } h \text { such that } A h \leq b \text { and } \\
\qquad\langle Q y, h-y\rangle>0 .
\end{gathered}
$$

Then the approximation

$$
R=\left\{v^{1}, v^{2}, \ldots, v^{m+1} \mid g\left(v^{i}\right)=0, i=1, \ldots, m+1\right\},
$$

where

$$
\begin{gathered}
v^{i}= \begin{cases}\mu_{i} Q^{-1} a^{i}, & \text { for } 1 \leq i \leq m, \\
\mu_{m+1} Q^{-1} d, & \text { for } i=m+1,\end{cases} \\
\mu_{m+1}=\left(\frac{2 \gamma}{\left\langle Q^{-1} d, d\right\rangle}\right)^{1 / 2}, \\
\mu_{i}=\left(\frac{2 \gamma}{\left\langle Q^{-1} a^{i}, a^{i}\right\rangle}\right)^{1 / 2}, \quad 1 \leq i \leq m,
\end{gathered}
$$

is a "resolving set" for problem (LRCP). 
Proof. According to Definition 3.3 one has to prove that if $z$ does not solve (LRCP), then $\max _{i}\left\{\left\langle\nabla g\left(w^{i}\right), u^{i}-w^{i}\right\rangle \mid i \in\{1,2, \ldots, m+1\}\right\}>0$.

So, suppose that $z$ is not a solution to $(L R C P)$, that is, there is $\bar{u}$ such that

$$
\begin{gathered}
\langle d, \bar{u}\rangle\langle\langle d, z\rangle, \\
A \bar{u} \leq b, \quad \frac{1}{2}\langle Q \bar{u}, \bar{u}\rangle-\gamma \geq 0 .
\end{gathered}
$$

We first show that there exists $u$ satisfying

$$
\begin{gathered}
\langle d, u\rangle<\langle d, z\rangle, \\
A u \leq b, \quad \frac{1}{2}\langle Q u, u\rangle-\gamma>0 .
\end{gathered}
$$

Because of (4.1), if

$$
A \bar{u} \leq b, \quad \frac{1}{2}\langle Q \bar{u}, \bar{u}\rangle-\gamma=0,
$$

then there is $h$ such that

$$
A h \leq b, \quad\langle Q \bar{u}, h-\bar{u}\rangle>0 .
$$

By the convexity of the polytope, $A h \leq b$ and $A \bar{u} \leq b$ imply that

$$
A u(\lambda) \leq b \quad \text { for each } \lambda \in[0,1]
$$

where $u(\lambda)=\lambda \bar{u}+(1-\lambda) h$.

For $\bar{\lambda}$ sufficiently close to 1 we obtain

$$
\langle d, u(\bar{\lambda})\rangle<\langle d, z\rangle .
$$

On the other hand, from assumption $(4.1)$ and the convexity of $g(\cdot)$ we have

$$
0<(1-\bar{\lambda})\langle\nabla g(\bar{u}), h-\bar{u}\rangle=\langle\nabla g(\bar{u}), u(\bar{\lambda})-\bar{u}\rangle \leq g(u(\bar{\lambda}))-g(\bar{u}) \text {. }
$$

We summarize the analysis as "for $u=u(\bar{\lambda})(4.6)$ holds for $\bar{\lambda}$ sufficiently close to $1 . "$

Let us now introduce the following notation:

$$
\begin{gathered}
a^{m+1}=d ; \\
\bar{A}^{\top}=\left(A^{\top}, a^{m+1}\right) ; \\
\bar{b}^{\top}=\left(b_{1}, \ldots, b_{m},\langle d, z\rangle\right) .
\end{gathered}
$$

We have $u$ satisfying (4.6). Then computing $\beta_{s}$ as

$$
\beta_{s}=\max \{\beta \mid \beta \geq 0, \bar{A}(\beta u) \leq \bar{b}\}
$$

we obtain point $u^{s}$ as $u^{s}=\beta_{s} u$. 
We assume without loss of generality that $\left\langle a^{s}, u^{s}\right\rangle=b_{s}$, that is, the sth constraint is active at $u^{s}$.

By the construction of the point $v^{s}$, since $\nabla g\left(v^{s}\right)=Q\left(\mu_{s} Q^{-1} a^{s}\right)=\mu_{s} a^{s}$, it is quite evident that $u^{s}$ solves the following problem (see (a2)):

$$
\begin{gathered}
\text { maximize }\left\langle\nabla g\left(v^{s}\right), x\right\rangle, \\
\text { subject to } \bar{A} x \leq \bar{b},
\end{gathered}
$$

corresponding to index $s$.

For the solution $u^{s}$ we can also solve the so-called level problem

$$
\begin{gathered}
\operatorname{maximize}\left\langle\nabla g(y), u^{s}-y\right\rangle, \\
\text { subject to } g(y)=0,
\end{gathered}
$$

analytically by the formula

$$
w^{s}=\alpha_{s} u^{s}, \quad \text { where } \alpha_{s}=\left(\frac{2 \gamma}{\left\langle Q u^{s}, u^{s}\right\rangle}\right)^{1 / 2}
$$

Because of the construction of $u^{s}$ and (4.6) we have

$$
g\left(u^{s}\right)=g\left(\beta_{s} u\right) \geq g(u)>0=g\left(w^{s}\right)
$$

which implies that $0<\alpha_{s}<1$.

Hence, due to positive definiteness of matrix $Q$ we have

$$
\begin{aligned}
\max _{i} & \left\{\left\langle\nabla g\left(w^{i}\right), u^{i}-w^{i}\right\rangle \mid i \in\{1,2, \ldots, m+1\}\right\} \\
\geq & \left\langle\nabla g\left(w^{s}\right), u^{s}-w^{s}\right\rangle=\left\langle Q w^{s}, u^{s}-w^{s}\right\rangle=\alpha_{s}\left(1-\alpha_{s}\right)\left\langle Q u^{s}, u^{s}\right\rangle>0,
\end{aligned}
$$

completing the proof.

\section{Concluding remarks}

In this paper, to establish optimality conditions for reverse convex problems, we have used the fact [17] that the inclusion $A \subset B$ is true for two convex sets $A, B \subset \mathbb{R}^{n}(B=\operatorname{cl}(B))$ if and only if

$$
N(B, y) \cap N(A, y) \neq \varnothing, \quad \forall y \in b d(B)
$$

rather than using the well-known equivalent conditions from convex analysis:

(i) $(B-y)^{\circ} \in(A-y)^{\circ}$ for $y \in(A \cap B)$;

(ii) $\sigma(y \mid A) \leq \sigma(y \mid B)$ for all $y \in \mathbb{R}^{n}$;

(iii) $N(B, y) \subset N(A, y)$ for all $y \in b d(B)$;

where $D^{\circ}, \sigma(\cdot \mid D), b d(D)$ are used for the polar, the support function, and the boundary of $D$, respectively.

In our case $A=F(z)$ and $B$ is the Lebesgue set of the reverse convex constraint function $g(\cdot)$. 
We also have touched on the question of how to prove that a level set approximation of the reverse convex constraint function is a "resolving set," which plays an essential role in providing convergence of the algorithm based on the optimality conditions obtained.

\section{Appendix}

\section{Theoretical method}

In this appendix, we provide the outline of a theoretical method for $(R P)$ and a sketch of the proof of its convergence [16]. For simplicity, in addition we assume that function $g(\cdot)$ is differentiable. In this case the optimality conditions (2.4), (2.14) could be written as

$$
\forall y: g(y)=0, \quad\langle\nabla g(y), x-y\rangle \leq 0, \quad \forall x \in F(z) .
$$

We now introduce the function $\varphi(\cdot)$ as follows:

$$
\varphi(z)=\sup _{x, y}\{\langle\nabla g(y), x-y\rangle \mid x \in S, f(x) \leq f(z), g(y)=g(z)\} .
$$

By the definition of "sup" we have

$$
\varphi(z) \geq 0, \quad \forall z \in S
$$

Moreover, for $z: g(z)=0$ it is not difficult to see that $\varphi(z)=0$ implies (A.1).

We also assume that

$$
\begin{gathered}
\text { for given } \varepsilon>0, \theta>0, \quad x \in S, g(x) \notin[-\theta, \varepsilon] \\
\text { there are } \mu>0, u=u(x) \in S \text { such that } \\
-\theta \leq g(u) \leq \varepsilon, \quad f(u) \leq f(x)-\mu g(x)+\varepsilon .
\end{gathered}
$$

Let $\left\{\varepsilon_{k}\right\},\left\{\theta_{k}\right\},\left\{\delta_{k}\right\}$ be numerical sequences satisfying

$$
\begin{gathered}
\varepsilon_{k}>\varepsilon_{k+1}>0, \quad \sum_{k=1}^{\infty} \varepsilon_{k}<+\infty, \quad \theta_{k}>0, \quad \delta_{k}>0, \\
\mu\left(\delta_{k}+\theta_{k}\right) \leq \varepsilon_{k}-\varepsilon_{k+1} .
\end{gathered}
$$

We are given $z^{k}$ such that $z^{k} \in S$ and $-\theta_{k} \leq g\left(z^{k}\right) \leq \varepsilon_{k}, k=0,1,2, \ldots$

By the construction of the function $\varphi(\cdot)$ according to the definition of "sup" for $\delta_{k}>0$ there are $p^{k}, x^{k}$ such that

$$
\begin{gathered}
\left\langle\nabla g\left(y^{k}\right), p^{k}-y^{k}\right\rangle \geq \varphi\left(z^{k}\right)-\delta_{k}, \\
g\left(p^{k}\right)=g\left(y^{k}\right), \quad p^{k} \in S, \quad f\left(p^{k}\right) \leq f\left(z^{k}\right) .
\end{gathered}
$$

By convexity of $g(\cdot)$ and $\varphi(\cdot) \geq 0$ we obtain

$$
-\delta_{k} \leq \varphi\left(p^{k}\right)-\delta_{k} \leq\left\langle\nabla g\left(y^{k}\right), p^{k}-y^{k}\right\rangle \leq g\left(p^{k}\right)-g\left(y^{k}\right) \leq g\left(p^{k}\right)+\theta_{k},
$$

which implies $g\left(p^{k}\right) \geq-\left(\delta_{k}+\theta_{k}\right)$. 
The method is defined by

$$
z^{k+1}= \begin{cases}p^{k}, & \text { if }-\theta_{k+1} \leq g\left(p^{k}\right) \leq \varepsilon_{k+1}, \\ u^{k}, & \text { if } g\left(p^{k}\right) \notin\left[-\theta_{k+1}, \varepsilon_{k+1}\right],\end{cases}
$$

where $u^{k} \in S$ is a vector that exists by assumption $(G)$ and satisfies

$$
-\theta_{k+1} \leq g\left(u^{k}\right) \leq \varepsilon_{k+1}, \quad f\left(u^{k}\right) \leq f\left(p^{k}\right)-\mu g\left(p^{k}\right)+\varepsilon_{k+1} .
$$

Theorem A.1. Suppose that assumptions (2.2), (A.4), (G) hold. Then a sequence $\left\{z^{k}\right\}$ generated by the method satisfies

$$
\lim _{k \rightarrow+\infty} \varphi\left(z^{k}\right)=0
$$

Proof. Because of (A.5), in the case of $z^{k+1}=p^{k}$ inequality $f\left(z^{k+1}\right) \leq f\left(z^{k}\right)$ holds. In the second case

$$
f\left(z^{k+1}\right) \leq f\left(p^{k}\right)-\mu g\left(p^{k}\right)+\varepsilon_{k+1} \leq f\left(z^{k}\right)+\mu\left(\delta_{k}+\theta_{k}\right)+\varepsilon_{k+1} .
$$

Hence, from (A.4) and the above two inequalities, we obtain

$$
f\left(z^{k+1}\right) \leq f\left(z^{k}\right)+\varepsilon_{k}, \quad k=0,1,2, \ldots
$$

When (A.11) and

$$
\varepsilon_{k}>\varepsilon_{k+1}>0, \quad \sum_{k=1}^{\infty}<+\infty
$$

hold, the numerical sequence $\left\{f\left(z^{k}\right)\right\}$ converges [16] and its limit is finite because of (2.2). Now, the index is divided into two parts $k_{s}, k_{t}$ :

$$
\begin{gathered}
-\theta_{k_{s}+1} \leq g\left(p^{k_{s}}\right) \leq \varepsilon_{k_{s}+1}, \\
-\left(\theta_{k_{t}+1}+\delta_{k_{t}}\right) \leq g\left(p^{k_{t}}\right) \leq \frac{1}{\mu}\left(f\left(z^{k_{t}}\right)-f\left(z^{k_{t}+1}\right)\right)+\varepsilon_{k_{t}+1} .
\end{gathered}
$$

Finally, we obtain $\lim _{k \rightarrow+\infty} g\left(p^{k}\right)=0$ which, in combination with inequalities (A.6), implies

$$
\lim _{k \rightarrow+\infty} \varphi\left(z^{k}\right)=0
$$

Theorem A.2. In addition to the conditions of Theorem A.1, suppose that (2.13) and the following condition:

$$
\exists v>0, \exists \rho>0, \quad\|\nabla g(y)\|>v, \quad \forall g(y) \geq-\rho
$$

are satisfied. Then an accumulation point of a sequence $\left\{z^{k}\right\}$ generated by the method solves problem $(R P)$. 
Proof. By construction we have

$$
-\theta_{k} \leq g\left(z^{k}\right) \leq \varepsilon_{k}
$$

which implies $\lim _{k \rightarrow+\infty} g\left(z^{k}\right)=0$. Using this fact and (2.13), together with $\lim _{k \rightarrow+\infty} \varphi\left(z^{k}\right)=$ 0 , we obtain the desired result from the sufficient optimality condition.

\section{Acknowledgments}

This article was completed during my stay at France Telecom R\&D. The author is indebted to France Telecom R\&D, particularly to J.-L. Lutton, T.-Q. Nguyen, and M. Bouhtou for their kind support. The author also would like to thank two anonymous referees for their valuable comments and suggestions which greatly improved the readability of the paper.

\section{References}

[1] P. P. Bansal and S. E. Jacobsen, Characterization of local solutions for a class of nonconvex programs, Journal of Optimization Theory and Applications 15 (1975), no. 5, 549-564.

[2] V. P. Bulatov, Approximation methods for solving some mathematical programming problems, Applied Mathematics, Irkutsk, SEI SB AS SSSR (1969), 10-15 (Russian).

[3] __ Embedding Methods in Optimization Problems, Izdat. "Nauka" Sibirsk. Otdel., Novosibirsk, 1977.

[4] R. Enkhbat, Algorithm for global maximization of convex functions over sets of special structures, Ph.D. thesis, State University, Irkutsk, 1990.

[5] D. Fortin and I. Tsevendorj, Piecewise-convex maximization problems: algorithm and computational experiments, Journal of Global Optimization 24 (2002), no. 1, 61-77.

[6] Gl_obal optimization and multi knapsack: a percolation algorithm, European Journal of Operational Research 154 (2004), no. 1, 46-56.

[7] R. J. Hillestad and S. E. Jacobsen, Reverse convex programming, Applied Mathematics and Optimization 6 (1980), no. 1, 63-78.

[8] R. Horst, P. M. Pardalos, and N. V. Thoai, Introduction to Global Optimization, Nonconvex Optimization and Its Applications, vol. 3, Kluwer Academic, Dordrecht, 1995.

[9] S. E. Jacobsen and K. Moshirvaziri, Computational experience using an edge search algorithm for linear reverse convex programs, Journal of Global Optimization 9 (1996), no. 2, 153-167.

[10] A. A. Kuznetsova, A. S. Strekalovskil̆, and I. Tsèvèèndorzh, On an approach to the solution of integer optimization problems, Zhurnal Vychislitel' noŭ Matematiki i Matematicheskoŭ Fiziki 39 (1999), no. 1, 9-16 (Russian), translation in Computational Mathematics and Mathematical Physics 39, no. 1, 6-13.

[11] R. Meyer, The validity of a family of optimization methods, SIAM Journal on Control and Optimization 8 (1970), no. 1, 41-54.

[12] J.-P. Penot, Duality for anticonvex programs, Journal of Global Optimization 19 (2001), no. 2, 163-182.

[13] A. S. Strekalovsky, Extremal problems on complements of convex sets, Kibernetika i Sistemnyı̆ Analiz 1993 (1993), no. 1, 113-126, translated in Cybernetics and Systems Analysis 29 (1993), no. $1,88-100$.

[14] Global optimality conditions for nonconvex optimization, Journal of Global Optimization 12 (1998), no. 4, 415-434.

[15] A. S. Strekalovsky and I. Tsevendorj, Testing the $\mathfrak{R}$-strategy for a reverse convex problem, Journal of Global Optimization 13 (1998), no. 1, 61-74. 


\section{Reverse convex problems}

[16] I. Tsevendorj, On searching global solution to reverse convex problems, Ph.D. thesis, State University, Irkutsk, 1997.

[17] _ Piecewise-convex maximization problems: global optimality conditions, Journal of Global Optimization 21 (2001), no. 1, 1-14.

[18] H. Tuy, Canonical DC programming problem: outer approximation methods revisited, Operations Research Letters 18 (1995), no. 2, 99-106.

[19] _ Convex Analysis and Global Optimization, Nonconvex Optimization and Its Applications, vol. 22, Kluwer Academic, Dordrecht, 1998.

[20] H. Tuy and L. T. Luc, A new approach to optimization under monotonic constraint, Journal of Global Optimization 18 (2000), no. 1, 1-15.

[21] A. B. Zalesski1̆, Nonconvexity of feasible domains and optimization of economic decisions, Èkonomika i Matematicheskie Metody 16 (1980), no. 6, 1069-1080 (Russian).

Ider Tseveendorj: PRiSM Laboratory, Computer Science Department, University of Versailles Saint Quentin en Yvelines, 45 avenue des Etats-Unis, 78035 Versailles Cedex, France

E-mail address: ider.tseveendorj@prism.uvsq.fr 


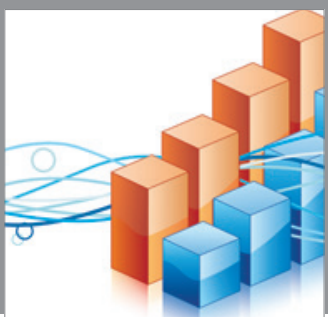

Advances in

Operations Research

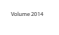

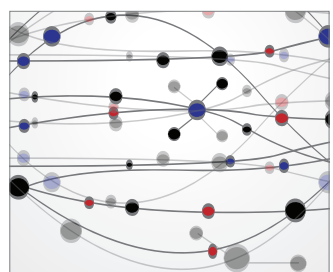

\section{The Scientific} World Journal
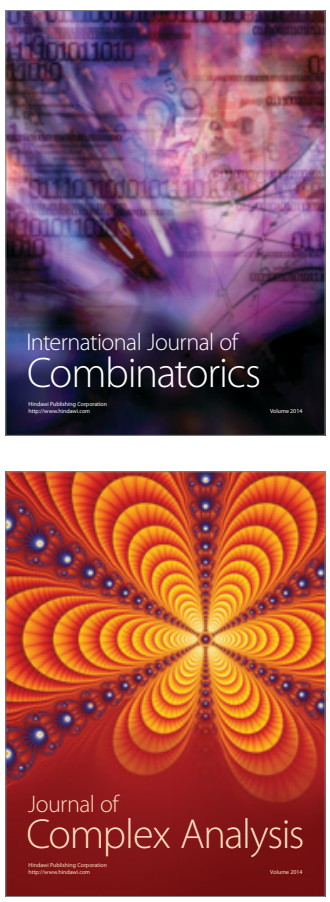

International Journal of

Mathematics and

Mathematical

Sciences
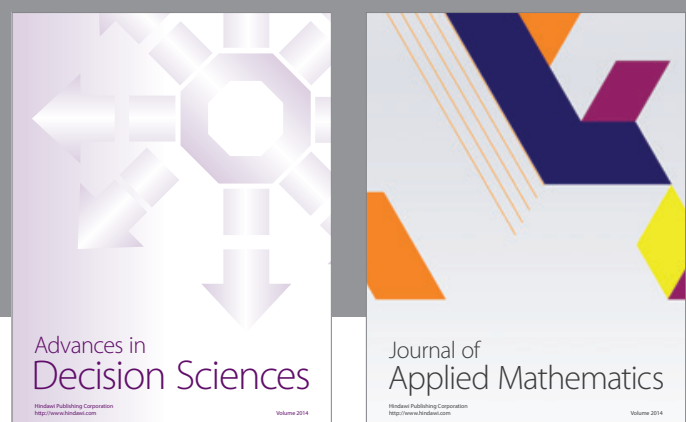

Journal of

Applied Mathematics
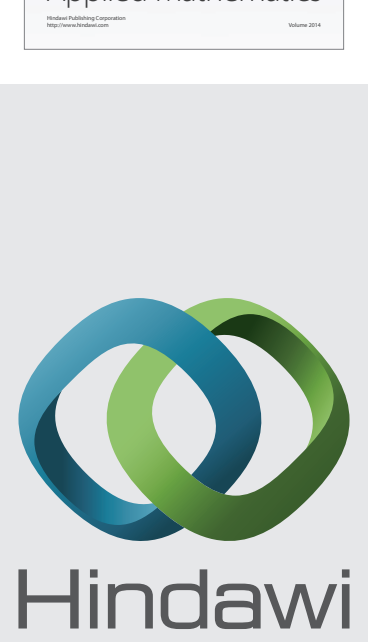

Submit your manuscripts at http://www.hindawi.com
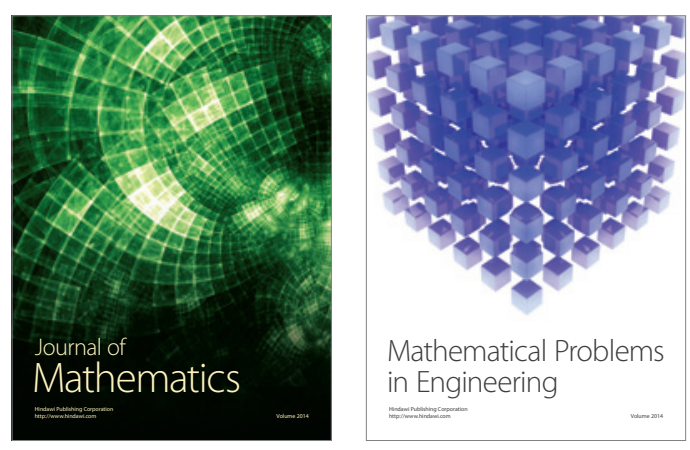

Mathematical Problems in Engineering
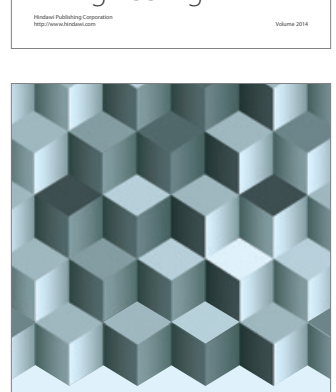

Journal of

Function Spaces
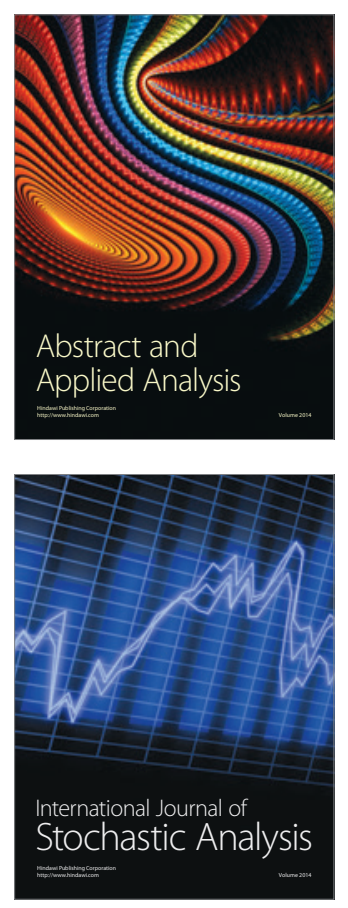

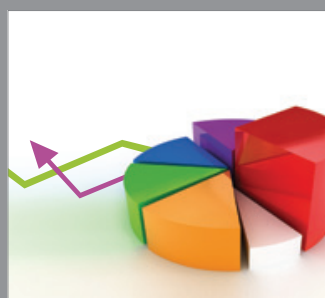

ournal of

Probability and Statistics

Promensencen
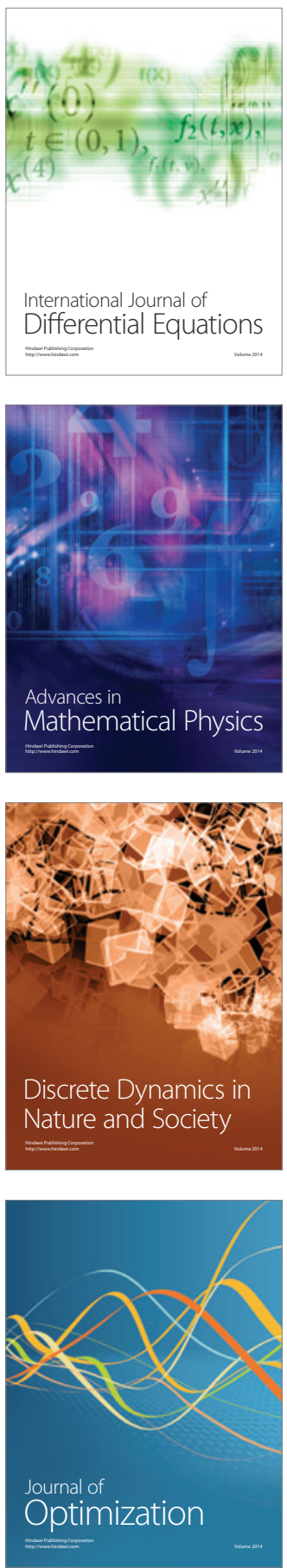